\title{
IMPACTS OF DIETARY SUPPLEMENTS AND NUTRIENT-RICH FOOD FOR PREGNANT WOMEN ON BIRTH WEIGHT IN SUGH EL-CHMIS /ALKHOMS - LIBYA
}

\author{
Alabed Ali A. Alabed1, Eman Ali Aljrbi², Abdullah Y. Al-Mahdi *3, Rasheed Abdulsalam", Anis Rageh Al- \\ Maleki ${ }^{5}$ and Hasanain Faisal Ghazi ${ }^{6}$ \\ 'Department of Community Medicine, Faculty of Medicine, Lincoln University College, 47301 Petaling Jaya, Selangor,
Malaysia \\ ${ }^{2}$ Department of Biotechnology, Faculty of Science, Lincoln University College, 47301, Petaling Jaya, Selangor, \\ Malaysia \\ ${ }^{3}$ Department of Microbiology, Faculty of Medicine, Lincoln University College, 47301, Petaling Jaya, Selangor, \\ Malaysia \\ ${ }^{4}$ Faculty of Dentistry, Lincoln University College, 47301, Petaling Jaya, Selangor, Malaysia \\ ${ }^{5}$ Medical Microbiology Department, Faculty of Medicine, University of Malaya, Kuala Lumpur, 50603, Malaysia \\ ${ }^{6}$ College of Nursing, Al- Bayan University, Baghdad, Iraq
}

${ }^{*}$ Corresponding author: Abdullah Y. Al-Mahdi

Email: dr.microbiology2019@gmail.com

\begin{abstract}
This study aims to determine the prevalence of low birth weight in Sugh El-Chmis /Alkhoms -Libya and the associated factors with the low birth weight of the baby. A cross-sectional study was performed in Libya, in 2015 using a pretested self-administered questionnaire on a stratified sample of 408 pregnant women. Descriptive, bivariate and multivariate analyses were carried out for statistical analysis. Out of a total of 408 respondents, the prevalence of low birth-weight baby was 23.5\%. The most of participants were aged between 19-26 years old (49\%). Dietary supplements (folic acid, iron, omega and vitamin $A, D, E, S, M$ ), natural fruits (apple, grape, watermelon, plum, figs, strawberry and peaches), all milk and dairy products (milk, cheese and yogurt), meat, fish, egg, legumes were significantly associated with baby weight $(P<0.05)$ using bivariate analysis. Furthermore, other factors such as culture and lifestyle and popular diets such as breakfast time, additional meal, soft drink, stimulants, barley wheat brown bread and dates also closely associated with the low-birth weight of new-borns $(P<0.05)$. The intake of dietary supplements and nutrient-rich food for pregnant women influenced on the birth weight in Sugh El-Chmis /AlkhomsLibya. Therefore, awareness regarding intake of dietary supplements and nutrient-rich food is highly recommended.
\end{abstract}

Keywords: Dietary supplementary, Nutrient-rich food, Libya, Low birth weight.

\section{INTRODUCTION}

Globally, more than 20 million infants representing $15.5 \%$ of all births, are born with low birth weight, $95.6 \%$ of them in developing countries. The level of low birth weight in developing countries (16.5\%) is more than double the level in developed regions (7\%). Half of all low-birth-weight babies are born in South-central Asia, where more than a quarter $(27 \%)$ of all infants weighs less than 2,500 gat births ${ }^{1}$.

Maternal nutrition and health are considered the most important regulator of human fetal growth. Improved maternal nutrition has been associated with increased fetal growth and a reduction in adverse birth outcomes in developing countries and populations with nutrient deficiencies ${ }^{2}$. However, if women are not well nourished, they are more likely to give birth to weak babies resulting in a high infant mortality rate. At birth, fetal weight is accepted as a single parameter that is directly related to the health and nutrition of the mother. On the other hand, it is an important determinant of the chances of the newborn to survive and experience healthy growth and development. This is because low birth weight is directly related to both immediate, long-term and very long-term development and well-being ${ }^{3}$.

World Health Organization (WHO) has defined low birth weight (LBW) as weight at birth of less than $2500 \mathrm{~g}^{4}$. This definition of LBW includes in its total a subgroup of infants who have suffered varying degrees of nutritional deprivation in utero. The latter subgroup, victims of intrauterine growth retardation (IUGR), constitutes the group at the highest degree of risk of both short-term and longterm complications. Fetal growth and birth weight are influenced by a variety of factors, racial, social and economic among others, as well as specific medical conditions that may be present or that may develop during pregnancy ${ }^{5}$.

A few investigations have demonstrated a relationship between anthropometric indicators and pregnancy outcomes. Both inadequate and unreasonable gestational weight gain is strongly connected with maternal-fetal complexities, for example, gestational diabetes, hypertensive pregnancy issue (HPD), macrosomia, and low birth weight ${ }^{6}$. Contrasted with ordinary weight, maternal overweight is identified with a higher 
danger of cesarean deliveries and a higher rate of aesthetic and postoperative complications in these deliveries. Low Apgar scores, macrosomia, and neural tube defects are more frequent in newborn children of fat mothers than in babies of ordinary weight moms ${ }^{7}$. Inadequacies in micronutrients may influence baby birth weight and survival, and poor vitamins. An intake builds the danger of maternal night visual deficiency ${ }^{8}$. Nutritional status in pregnancy is a basic issue that can't be neglected while considering the wellbeing of a nation, particularly the developing. A woman's dietary status has essential ramifications for her well-being and the well-being of her kids. Malnutrition in women brings about lessened efficiency, an expanded weakness to contaminations, moderate recuperation from sickness, and elevated dangers of unfriendly pregnancy results. For instance, a woman who has poor nutritious status, as shown by a low body mass index (BMI), short stature, or other micronutrient inadequacies, has more danger of deterred work, of having an infant with low birth weight, of delivering lower quality breast milk, of passing on from baby blues discharge, and of contracting maladies alongside her infant ${ }^{9}$. The brilliant interim for mediation stretches out from pregnancy to two years old. On the off chance that undernutrition isn't tended to amid these critical times of life, it might cause irreversible harm for future improvement towards adulthood, hence expanding the danger of young ladies getting to be malnourished moms, who at that point have a low-birth-weight infant.

The percentage and number of low-birth-weight infants by UNICEF was published in 2000 , showed that $15 \%$ was estimated of low birth weight in the Middle East and North Africa with $60 \%$ of birth not weighed. The same result was reported for Western and Central Africa and a little less in Eastern and Southern Africa by 14\%. In Libya, it was almost half by $7 \%$ of low-birth-weight infants for $1000^{1}$. Moreover, stressful life events experienced by pregnant women may lead to adverse obstetric outcomes. A study in Benghazi compared the rates of preterm, low-birth-weight and cesarean-section births at Al-Jamhouria hospital in the months before and during the armed conflict in Libya in 2011. Compared with the preceding months there was a significant rise during the conflict in the rate of deliveries involving preterm $(3.6 \%$ versus $2.5 \%)$ and lowbirth-weight $(10.1 \%$ versus $8.5 \%)$ infants and cesarean sections (26.9\% versus $25.3 \%$ ).

According to the most recent WHO data from 2018, low birth weight fatalities in Libya totaled 389 , accounting for 1.24 percent of all deaths. Libya ranks at the 109 positions in the world with an age-adjusted death rate of 5.48 per 100,000 people. Between 1995 and 2014, the prevalence of underweight, weight for age, male (percentage of children under the age of 5) in Libya increased significantly from 4.6 to $13 \%$, climbing at an annual rate of 106.35 percent. Therefore, this study aims to determine the prevalence of low birth weight in Sugh El-Chmis / Alkhoms -Libya and the associated factors with the birth weight of the baby.

\section{MATERIALS \& METHODS}

\section{Study design and sampling}

A cross-sectional study was conducted among pregnant women who were in their first, second or third trimester visiting Women and Children government hospital in Sugh El-Chmis, Libya. The pregnant women who used to take dietary supplements and natural food during their pregnancy were chosen to participate in this study. People with the ability to read and write the questionnaire considered eligible for this study. Participating in this study was voluntary and random. A total of 408 questionnaires were collected. All respondents were briefed about the study at the beginning of the questionnaire as a short introduction. The questionnaires were given to pregnant women after obtained verbal consent from them. Only those who fulfilled the inclusion and exclusion criteria and consented were included in this study. The data excel file was obtained by the authors after it was taken from the Google form.

\section{Measurement tool}

A primary questionnaire was developed after a comprehensive literature review and validated by a panel of experts consisting of an epidemiologist, nutrient specialist, public health specialist and biostatistician to be used for pregnant women who visiting Women and Children Government Hospital at Sugh El-Chmis, Libya. A pre-test was performed at ALMGREF health center, Libya to make sure the questions are clear and easy to understand. After the approval of the pre-testing, the survey was performed via a questionnaire. The questions were prepared in the Arabic language and back-translated into the English language. The questionnaires were divided into five sections including economic and social situation; dietary supplements; natural food; culture and lifestyle and popular diets; and birth weight and maternal and child health status. Most of the items were designed to be closed-ended questions by answering yes or no. The questionnaire was designed to be anonymous, and informed consent was obtained from every respondent as it was sent along with the survey. The data were kept confidential and the results did not identify the respondents personally. Participating in the research was voluntary and anonymous.

\section{Data analysis}

Data analysis was performed using Statistical Package for Social Sciences (SPSS) version 22 
(Chicago, IL, USA). Continuous variables were expressed as mean \pm standard deviation (SD) while categorical variables were presented as frequency and percentage. Descriptive statistics were carried out to determine the socio-demographic factors and baby weight. Inferential statistics (chi-square test) was used to assess the association between socio-demographic factors and baby weight. Then, multivariate analysis was performed to identify the most important factors associated with baby weight. Multiple logistic regression was used for this analysis. The significance level was set at $(P \leq 0.05)$ for all statistical procedures.

\section{RESULTS}

\section{Socio-Demographic Data}

Out of total of 408 participants in this study, the prevalence of low birth was $23.5 \%$. The most of respondents were aged between 19-26 years old (49\%), $112(27.5 \%)$ aged between $27-36$ years old, $72(17.6 \%)$ aged more than 36 years old and 24 $(5.9 \%)$ aged less than 19 years old. Women with less than 5 years of marriage were the majority by $208(51.0 \%)$ compared to other groups which represent $40(9.8 \%)$ for $5-10$ years marriage, 72 (17.6\%) for $10-15$ years marriage and $8821.6 \%$ for more than 15 years marriage. The majority of the respondents were university-educated with 232 (56.9\%) while intermediate; and read and write accounted for 144 (35.3\%) and 32 (7.8\%) respectively. It was found that most of women 256 $(62.7 \%)$ were from family with middle income 501 1000 LYD compared to $112(27.5 \%)$ with less than 500 LYD, 32 (7.8\%) with 1011-1500 LYD and 8 $(2.0 \%)$ with more than 1500 LYD. The participants were mostly housewives by $200(49.0 \%)$ followed by $16(39.2 \%)$ teachers, $40(9.8 \%)$ employees and $8(2.0 \%)$ craftswomen. Finally, $248(60.8 \%)$ women had 2-5 children compared to $88(21.6 \%)$ one child and $72(17.6 \%) 6-9$ children. The summary of the characteristics is shown in Table 1.

\section{Bivariate Analysis Related to Dietary Supplements}

Chi-square test was used to determine the association between baby weight and all the categorical variables such as: dietary supplements, natural food, meat, eggs and legumes, culture and lifestyle and popular diets and maternal and child health status. It was found that dietary supplements (folic acid, iron, omega and vitamin A, D, E, S, M) were significantly associated with baby weight $(P<0.05)$. Only calcium was not statistically significant with baby weight $(\mathrm{P}>0.05)$. For vegetables, it was demonstrated that tomato, beef and cabbage were significantly associated with baby weight $(P$ $<0.05$ ) except lettuce, cucumber and carrots.

For natural fruits, it was revealed that apple, grape, watermelon, plum, figs, strawberry and peaches were significantly associated with baby weight $(P<0.05)$. On the other hand, banana, apricot, tin pear, orange and kiwi were not statistically significant with baby weight $(P>0.05)$. Interestingly, it was showed that all the dairy products (milk, cheese and yogurt), red meat, white meat, fish, eggs and all kind of legumes were significantly associated with baby weight $(P$ $<0.05$ ). Furthermore, it was demonstrated that all factors for culture and lifestyle and popular diets such as breakfast time, additional meal, soft drink, stimulants, barley wheat brown bread and dates were too significantly associated with baby weight $(P<0.05)$. The analysis indicated that newborn health status, mother health, delivery date and breast feeding were statistically associated with baby weight $(P<0.001)$ but only delivery status factor was not significant with baby weight $(P>0.05)$.

\section{Multivariate analysis}

Multiple logistic regression analysis was performed to estimate the predictive risk factors of baby weight when other confounders were controlled or adjusted so that they could be calculated. All the variables with a total sample size of 408 , which were statistically significant in the bivariate analysis, were included in the multiple logistic regression analysis. The dependent variable that measures the baby weight was YES, which was coded as (l) for normal weight, while NO was coded as (0) for not normal.

As shown in Table 3, logistic regression analysis revealed that the factors that were significantly associated with baby weight after controlling the confounders $(P<0.05)$ by using stepwise forward LR logistic regression method. The results show that the dates and breastfeeding $(P<0.001)$ made a significant contribution to prediction, whereas other variables were not. The most important factor responsible for the estimation of the baby weight was identified by using the Wald criterion, which gave the 'importance' of the contribution of each variable in the model. The Wald test demonstrated that the dates was the most important predictor for baby weight estimation. 
Table 1: Descriptive analysis related to demographical factors

\begin{tabular}{lcc}
\hline Variables & Frequency & Percent \\
\hline Age & 24 & \\
Less than 19 & 200 & 49.9 \\
19-26 & 112 & 27.5 \\
27-36 & 72 & 17.6 \\
$\quad$ More than 36 & 208 & \\
Duration of marriage & 40 & 51.0 \\
$\quad$ Less than 5 & 72 & 9.8 \\
5-10 & 88 & 17.6 \\
10-15 & & 21.6 \\
More than 15 & 144 & 35.3 \\
Educational level & 232 & 56.9 \\
Intermediate educ. & 32 & 7.8 \\
University educ. & 112 & 27.5 \\
Read and write & 256 & 62.7 \\
Family income & 32 & 7.8 \\
Less than 500 LYD & 8 & 2.0 \\
501-1000 & & \\
1001-1500 & 200 & 49.0 \\
More than 1500 LYD & 40 & 9.8 \\
Occupation & 160 & 39.2 \\
Housewife & 8 & 2.0 \\
Employee & & \\
Teacher & 88 & 21.6 \\
Craftswomen & 248 & 60.8 \\
Nof children & 72 & 17.6 \\
One child & 408 & 100.0 \\
2-5 & & \\
6-9 & & \\
TOTAL & &
\end{tabular}

Table 2a: Bivariate analysis for most important factors related to baby low birth weight

\begin{tabular}{lcc}
\hline Variables & $\mathbf{X}^{2}$ test & P value \\
\hline Dietary supplementary & & \\
\hline Folic acid & 17.018 & $0.002^{*}$ \\
Calcium & 6.613 & 0.307 \\
Iron & 28.714 & $0.000^{*}$ \\
Omega & 23.099 & $0.000^{*}$ \\
Vit. A, D, E, S, M & 12.669 & $0.013^{*}$ \\
Vegetables & & \\
Lettuce & 2.931 & 0.401 \\
Tomatoes & 15.551 & $0.019^{*}$ \\
Cucumber & 6.221 & 0.101 \\
Beet & 50.158 & $0.000^{*}$ \\
Carrots & 4.670 & 0.198 \\
Cabbage & 49.244 & $0.000^{*}$ \\
Fruits & & \\
Apple & 54.697 & $0.000^{*}$ \\
Banana & 4.222 & 0.238 \\
Grape & 17.468 & $0.001^{*}$ \\
Watermelon & 10.440 & $0.015^{*}$ \\
Apricot & 3.193 & 0.363 \\
Tin pear & 7.553 & $0.056^{*}$ \\
Plum & 11.342 & $0.010^{*}$ \\
Figs & 13.293 & $0.004^{*}$ \\
Orange & 7.172 & 0.067 \\
Kiwi & 6.417 & 0.093 \\
Strawberry & 32.579 & $0.000^{*}$ \\
Peaches & 11.613 & $0.009^{*}$ \\
\hline
\end{tabular}


Table 2b: Bivariate analysis for most important factors related to baby low birth weight

\begin{tabular}{|c|c|c|}
\hline Variables & $\mathrm{X}^{2}$ test & $\mathrm{P}$ value \\
\hline \multicolumn{3}{|l|}{ Milk and dairy products } \\
\hline$\overline{M i l k}$ & 28.071 & $0.000^{*}$ \\
\hline Cheese & 24.360 & $0.000^{*}$ \\
\hline Yogurt & 66.771 & $0.000^{*}$ \\
\hline \multicolumn{3}{|l|}{ Meat, fish, eggs and legumes } \\
\hline Red meat & 64.099 & $0.000^{*}$ \\
\hline White meat & 58.820 & $0.000^{*}$ \\
\hline Fish & 8.171 & $0.029 *$ \\
\hline Eggs & 22.789 & $0.003^{*}$ \\
\hline All kind of legumes & 22.280 & $0.001^{*}$ \\
\hline \multicolumn{3}{|l|}{ Culture, lifestyle and popular } \\
\hline$\overline{\text { Breakfast time }}$ & 40.917 & $0.000^{*}$ \\
\hline Additional meal & 66.413 & $0.000^{*}$ \\
\hline Soft drink & 30.775 & $0.000^{*}$ \\
\hline Stimulants & 26.673 & $0.000^{*}$ \\
\hline $\begin{array}{l}\text { Meal by barley wheat brown } \\
\text { bread }\end{array}$ & 69.424 & $0.000^{*}$ \\
\hline Dates & 89.747 & $0.000^{*}$ \\
\hline \multicolumn{3}{|l|}{ Maternal and child health } \\
\hline$\overline{\text { Newborn health status }}$ & 8.938 & $0.011^{*}$ \\
\hline Mother health & 23.050 & $0.000^{*}$ \\
\hline Delivery date & 16.299 & $0.001^{*}$ \\
\hline Delivery status & 0.090 & 0.887 \\
\hline Breast feeding & 37.430 & $0.000^{*}$ \\
\hline
\end{tabular}
${ }^{*} p<0.05$

Table 3: Multivariate Analysis for Most Important Factors Related to Baby Low Birth Weight

\begin{tabular}{|c|c|c|c|c|c|c|c|c|}
\hline \multirow[b]{2}{*}{ Variables } & \multirow[b]{2}{*}{ B } & \multirow[b]{2}{*}{ S.E. } & \multirow[b]{2}{*}{ Wald } & \multirow[b]{2}{*}{ df } & \multirow[b]{2}{*}{ Sig. } & \multirow[b]{2}{*}{$\operatorname{Exp}(B)$} & \multicolumn{2}{|c|}{ 95\% C.I. for EXP(B) } \\
\hline & & & & & & & Lower & Upper \\
\hline Dates & & & 93.157 & 3 & $0.000^{*}$ & & & \\
\hline Daily & 3.014 & 0.417 & 52.298 & 1 & $0.000^{*}$ & 20.363 & 8.997 & 46.086 \\
\hline 2-3 times a week & 1.275 & 0.237 & 28.923 & 1 & $0.000^{*}$ & 3.579 & 2.249 & 5.695 \\
\hline 3-4 weeks & 4.152 & 0.553 & 56.410 & 1 & $0.000^{*}$ & 63.536 & 21.503 & 187.727 \\
\hline Breastfeeding & & & 50.357 & 2 & $0.000^{*}$ & & & \\
\hline Normal & -0.440 & 0.255 & 2.975 & 1 & 0.085 & 0.644 & 0.391 & 10.062 \\
\hline Non -normal & -3.673 & 0.518 & 50.353 & 1 & $0.000^{*}$ & 0.025 & 0.009 & 0.070 \\
\hline
\end{tabular}

\section{DISCUSSION}

To the best of our knowledge, this is the first time that a study demonstrates the prevalence of low birth weight and their associated factors in Sugh El-Chmis, Libya. The findings can be considered the first step in providing data on low birth weight and will help in creating awareness among Libya pregnant women, to provide insight in designing future action steps to bridge the gaps as an effort against low birth weight. In this study, we found that there was a relationship between taking protein day by day and newborn childbirth weight $(p<0.05)$. This outcome was coordinated with the investigation of Whitney et al. (2007) study which demonstrated that protein is important for the fast fetal development, the extension of the uterus, mammary organs, and placenta, increments in maternal blood volume, development of amniotic liquid, and capacity save for work, conveyance, and lactation ${ }^{10}$. Many shreds of evidence have shown that macronutrients (protein) supplementation were associated with an increase in maternal weight and mean birth weight and a decrease in the number of low birth weight babies. According to the World Health Organization (1995), an additional $100 \mathrm{kcal} /$ day throughout the pregnancy can increase birth weight by about $100 \mathrm{~g}$ and the risk of IUGR would be halved for undernourished women ${ }^{11}$.

Poor iron status may affect immune function and thus increase host susceptibility to "genital tract infection" and also increase oxidative stress of the placenta. Low hemoglobin status causes chronic 
hypoxia ${ }^{12}$. Supplementation of $60 \mathrm{mg}$ of ferrous iron and $0.4 \mathrm{mg}$ of folic acid /day significantly increased the birth weight. To obtain a maximum effect on birth weight iron supplementation should be started in early pregnancy ${ }^{13}$. Iron and folic acid deficiency in early pregnancy are associated with neurological defects. In this study, it proved that iron has an association with baby weight $(p<0.05)$ and supported the results from Muthayya et al. (2006) study ${ }^{13}$. Prevalence of anemia among pregnant women in Libya is comparable to that of a severe public health concern, indicating that anemia in pregnant women is a public health issue in Libya. According to Elzahaf and Omar (2016) study, it has been reported that anaemia was more common in pregnant women aged $15-20$ to $36-40$ years old and with only a primary school education. Anemia was found in $\mathbf{5 4 . 6}$ percent of pregnant women, with 44.5 percent having mild anemia. The third trimester of pregnancy has a higher prevalence of anemia (59.6 percent). Thus, awareness regarding regular intake of iron and folic acid tablet was highly recommended in their study. Furthermore, vitamin $A$ is fundamental for development, and the separation of most cells and tissues. It is essential for sound fetal advancement. Pregnant women are by and large encouraged to expand their vitamin intake by $10-40 \%{ }^{14}$.

Calcium is important because it aids to strengthen the baby's rapidly-developing bones and teeth, and boosts muscle and heart. It plays a vital role in nerve development too. Moreover, it is still essential for teeth and bones of a pregnant woman. If the pregnant woman doesn't get enough calcium in their diet, the body will take what the baby needs. It is especially the case during the third semester when bone development peaks at 250-250 mg transferred from the pregnant woman and the baby every day ${ }^{15}$. A woman who is not getting enough calcium during her pregnancy makes her more susceptible to osteoporosis. Unlike our study demonstrated that calcium was not statistically significant $(p=$ 0.307). However, concerning the dietary supplements, this investigation shows that there was a factual huge positive relationship between taking supplements (eg. omega and vitamins) and new-born childbirth weight. This outcome was bolstered by Lassi et al. (2006) study ${ }^{16}$. They found that numerous micronutrient supplementations brought about a measurably diminished number of low-birth-weight babies, small for gestational age (SGA) babies and maternal pallor.

Omega- 3 fatty acids are essential and can only be obtained from the diet. Nevertheless, a great many people, including pregnant women, are not getting sufficiently omega-3 from their eating regimen. Omega-3 unsaturated fats are basic amid pregnancy, particularly the long-chain omega- 3 unsaturated fats DHA and EPA. These are found in high sums in fish and help assemble the cerebrum and eyes of the baby. Pregnant women are encouraged to confine their fish intake for twice per week. Due to the mercury and different contaminants found in greasy fish, this has made a few women maintain a strategic distance from fish by and large, in this way restricting the intake of fundamental omega-3 unsaturated fats. Nonetheless, studies have demonstrated that pregnant women who eat 2-3 dinners of greasy fish every week accomplish the suggested intake of omega-3 and increment their blood levels of EPA and DHA. Salmon is likewise one of not very many common wellsprings of vitamin $D$, which is regularly ailing in the eating routine. It is imperative for some procedures in the body, including bone wellbeing and resistant capacity ${ }^{17}$. In our study, it revealed that omega was significantly associated with low birth weight $(p=0.000)$. Based on Albakoush and Azab (2019) study, it has been demonstrated that pregnant women in Western-Libya were at an increased risk of vitamin D insufficiency. Pregnant women's vitamin $D$ levels were found to be much lower than non-pregnant women's. Vitamin D levels were below $20 \mathrm{ng} / \mathrm{ml}$ in 84.8 percent of the study population, while calcium levels were below 8.4 $\mathrm{mg} / \mathrm{dl}$ in $46.8 \%$. Vitamin $\mathrm{D}$ levels were less than $20 \mathrm{ng} / \mathrm{ml}$ in 95.2 percent, 91.2 percent, and 66.7 percent of pregnant women in the first, second, and third trimesters, respectively. Calcium levels were less than $8.4 \mathrm{mg} / \mathrm{dl}$ in 61.9 percent, 35.3 percent, and 50 percent of pregnant women in the first, second, and third trimesters, respectively. The study focused on maternal nutrition, particularly optimal vitamin D and calcium intake, which may prepare the way for future bone health concerns such as osteoporosis prevention in the long term. Moreover, vegetables and fruits are one of the most important nutrients that should be taken by pregnant women as suggested by the World Health Organization (WHO). Fresh vegetables and fruits contain lots of essential vitamins and nutrients and are a good source of fiber too. This not only helps the fetal development but also keep the pregnant woman body in the best condition possible. In this study, the importance of these nutrients was very clear through our results which demonstrated that most of them were statistically significant and have an association with baby weight $(p<0.05)$.

Red and white meat is a great source of protein, zinc and iron. These are essential nutrients that every pregnant woman should consume in adequate amounts during pregnancy. Expanding fish was more marginally among the mothers who brought forth LBW new-born children. This implies that there is a negative connection between the utilization of fish amid pregnancy and baby birth weight. This was due to the defilement with toxins and dangerous metals as mercury which might be kept in angle. This toxic compound may gather in the circulation system after some time 
and could harm the new-born child developing cerebrum and sensory system. This is conflicting with Thorsdottir et al. (2004) study which discovered the baby of women in the most reduced amounts of fish utilization weighed less, were shorter and had a littler head outline during childbirth than those of women expending higher measures of fish $^{18}$. Legumes are brilliant plantbased sources of fiber, protein, press, folate (B9) and calcium, all of which the body needs a greater amount of amid pregnancy. Folate is one of the $B$ vitamins (B9). It is imperative for the wellbeing of the mother and baby, particularly amid the primary trimester ${ }^{19}$. Eggs are rich in the mineral choline and Omega 3 fatty acids. They are important for baby's brain development. It helps to prevent neural tube defects in vitro as well.

In this study, culture, lifestyle and popular diets (soft drink, stimulants, dates and barley, wheat brown bread) significantly associated with low birth weight. According to Abubakari and Jahn (2016) study, skipping breakfast affects fetal development. Soft drinks have carbon dioxide bubbles $^{20}$. Carbonic acids will cause severe heartburn and indigestion, increasing the acidic levels in a pregnant lady. Barley wheat brown bread is a good source of the $B$ vitamins (including B1, B2, folic acid, and niacin) which is crucial for baby development and growth of the placenta. Based on Karen Miles (2020) study, it has been demonstrated that the recommended amount of fiber during pregnancy is $28 \mathrm{~g}$ a day, which helps prevent constipation and haemorrhoids ${ }^{21}$.

Finally, dates are guaranteed as 'heart solid nourishments' by the American Heart Association because of their low cholesterol and immersed fat substance. They contain supplements that are fundamental to the baby's sound development and advancement. They have many advantages, for example, insurance of the liver, help vitality, enhances weight, support by insusceptible framework, enhances hemoglobin, enhances vision, cures looseness of the bowels and numerous others ${ }^{22}$. In this study, date one the most important and predictors for baby weight as it was statistically significant in the bivariate analysis and multivariate analysis as well along together with breastfeeding $(p<0.05)$. According to the World Health Organization (2020), breastfeeding has many health benefits for both the mother and baby ${ }^{23}$. Breast milk contains all the nutrients a baby needs in the first six months of life. It protects against diarrhoea, allergies, sickness, obesity and infections.

\section{CONCLUSION}

The present findings highlight concerns regarding the intake of dietary supplements (folic acid, iron, omega and vitamin $A, D, E, S, M)$ ), natural fruits (apple, grape, watermelon, plum, figs, strawberry and peaches), milk and dairy products (milk, cheese and yogurt), meat, fish, egg, legumes, influence the low birth weight of new-borns in Sugh El-Chmis /Alkhoms-Libya. Furthermore, other factors such as culture and lifestyle and popular diets such as breakfast time, additional meal, soft drink, stimulants, barley wheat brown bread and dates also causes the low-birth weight of new-borns. These findings will aid to design effective interventions to overcome the low birth weight and create a more robust awareness among pregnant women.

\section{Competing Interests}

The authors have declared that no competing interest exists.

\section{REFERENCES}

1. United Nations Children's Fund and World Health Organization, Low Birthweight: Country, regional and global estimates. UNICEF, New York, 2004.

2. Black RE, Victora CG, Walker SP, et al. Maternal and child undernutrition and overweight in low-income and middleincome countries. The lancet. 2013; 382(9890):427-51.

3. Agarwal K, Agarwal A, Agrawal VK, et al. Prevalence and determinants of "low birth weight" among institutional deliveries. Annals of Nigerian Medicine. 2011; 5(2):48.

4. WHO. Global nutrition targets 2025: low birth weight policy brief Geneva. World Health Organization; 2014. Available from:

https://www.who.int/nutrition/publica tions/globaltargets2025_policybrief_lb w/en/.

5. Hoyme HE, May PA, Kalberg WO, et al. A practical clinical approach to diagnosis of fetal alcohol spectrum disorders: clarification of the 1996 institute of medicine criteria. Pediatrics. 2005; 115(1):39-47.

6. Geraci M. Estimation of regression quantiles in complex surveys with data missing at random: An application to birthweight determinants. Statistical methods in medical research. 2016; 25(4):1393-421.

7. Gunatilake RP, Perlow JH. Obesity and pregnancy: clinical management of the obese gravida. American journal of obstetrics and gynecology. 2011; 204(2):106-19. 
8. World Health Organization. Vitamin A supplementation in postpartum women. 2009. Available from: https: / /www.who.int/elena/titles/vita mina_postpartum/en/

9. Imdad A, Lassi Z, Salaam R, et al. Prenatal nutrition and nutrition in pregnancy: Effects on long-term growth and development. InEarly Nutrition and Long-Term Health 2017 Jan 1 (pp. 324). Woodhead Publishing.

10. Whitney E, Rolfes SR. 2002. Understanding Nutrition. Ninth Edition Belmont: Wadsworth Com.

11. World Health Organization, Maternal anthropometry and pregnancy outcomes. Bull. World Health Org.1995; 73(supll):1-98.

12. Murphy JD, Aronovitz MJ, Reid LM. Effects of chronic in utero hypoxia on the pulmonary vasculature of the newborn guinea pig. Pediatric research. 1986; 20(4):292-5.

13. Muthayya S, Kurpad AV, Duggan CP, et al. Low maternal vitamin B 12 status is associated with intrauterine growth retardation in urban South Indians. European journal of clinical nutrition. 2006; 60(6):791-801.

14. Beaton GH. Effectiveness of vitamin A supplementation in the control of young child morbidity and mortality in developing countries. United Nations Subcommittee on Nutrition (SCN) News. 1993; 9:17-23.

15. Karla Walsh. How Much Calcium Do You Need During Pregnancy? (2020). Available

from:https: / /www. whattoexpect.com/ pregnancy/diet/calcium-during pregnancy/\#: :text=Calcium\%20helps\%2 Ostrengthen\%20your\%20baby's, take\%20w hat\%20your\%20baby\%20needs.

16. Lassi ZS, Salam RA, Haider BA, et al. Folic acid supplementation during pregnancy for maternal health and pregnancy outcomes. Cochrane Database of Systematic Reviews. 2013(3).

17. Calder PC. Omega-3 polyunsaturated fatty acids and inflammatory processes: nutrition or pharmacology? British journal of clinical pharmacology. 2013; 75(3):645-62.
18. Thorsdottir I, Birgisdottir BE, Halldorsdottir S, et al. Association of fish and fish liver oil intake in pregnancy with infant size at birth among women of normal weight before pregnancy in a fishing community. American journal of epidemiology. 2004; 160(5):460-5.

19. Hjertholm KG, Iversen PO, HolmboeOttesen G, et al. Maternal dietary intake during pregnancy and its association to birth size in rural Malawi: A crosssectional study. Maternal \& child nutrition. 2018; 14(1):e12433.

20. Abubakari A, Jahn A. Maternal dietary patterns and practices and birth weight in Northern Ghana. PLoS One. 2016; 11(9):e0162285.

21. Karen Miles. Grains in your pregnancy diet. (2020). Available from: https: / /www.babycenter.com/0_grains -in-your-pregnancy-diet_1497.bc

22. Jadidi MY, Sang SJ, Lari H. The effect of date fruit consumption on spontaneous labor. Journal of Pizhūhish dar dīn va salāmat. 2016; 1(3):4-10.

23. World Health Organization 2020. Available from; https: / /www.who.int/elena/titles/excl usive_breastfeeding/en/ 